\title{
Aromatic Amines
}

National Cancer Institute

\section{Source}

National Cancer Institute. Aromatic Amines. NCI Thesaurus. Code C1815.

Amine having a univalent organic group (as phenyl) derived from an aromatic

hydrocarbon by the removal of one hydrogen atom. (ISV) 